\title{
Reversibilidad mandibular de la rigidez cadavérica por medios químicos en un modelo de rata
}

\author{
Jairo Alfonso Fovar Franeo ${ }_{\perp}^{1 *}$, Diana Constanza Aparieio Castellanos ${ }_{\perp}^{2}$, Luisa Fernanda Henríquez Higuera ${ }_{\perp}^{2}$ \\ ${ }^{1}$ Departamento de Nutrición y Bioquímica. \\ ${ }^{2}$ Especialización en Odontología Legal y Forense, Departamento de Odontología. \\ Pontificia Universidad Javeriana. Cra. $7^{a}$ \# 43-82, Bogotá D.C. Colombia \\ *jatovar@javeriana.edu.co
}

Recibido: 08-04-2008; Aceptado: 05-06-2009

\begin{abstract}
Resumen
El principal problema que recae sobre el odontólogo en la toma de evidencias post mortem es la apertura bucal, limitada por el fenómeno de rigidez cadavérica el cual inicia a las 3 horas después de la muerte, siendo éste un fenómeno que persiste hasta ser destruido por los procesos autolíticos tardíos de descomposición, a partir de las 36 horas post mortem. Objetivo. Se estudió la posibilidad de acelerar la reversión de la rigidez cadavérica mandibular por medio de sustancias químicas para facilitar la apertura bucal. Materiales y métodos. Se encaminó al uso de sustancias que produzcan la alteración del pH, quelación del calcio intramuscular o proteólisis del complejo actina-miosina de los músculos masticatorios que presenten rigidez cadavérica en ratas Wistar. Resultados. Se determinó que el tiempo de establecimiento de la rigidez cadavérica mandibular en el modelo de rata, fue de 2,5 horas. A las 3,5 horas, una vez establecida la rigidez, se realizaron infiltraciones con EDTA $(20 \mathrm{mM}), \mathrm{NaHCO}_{3}(50 \mu \mathrm{M}), \mathrm{Na}_{2} \mathrm{CO}_{3}(50 \mu \mathrm{M})$ y papaína $(10 \mu \mathrm{M})$ encontrándose que las soluciones de $\mathrm{NaHCO}_{3}$ y $\mathrm{Na}_{2} \mathrm{CO}_{3}$ incrementaron significativamente $(\mathrm{p}<0,05)$ la velocidad de reversión $(\mathrm{mm} / \mathrm{h})$ desde las $5 \mathrm{~h}$, en un $108 \%$ y un $100 \%$, respectivamente. A partir de este ensayo se duplicó la concentración de $\mathrm{NaHCO}_{3}$ y se preparó una mezcla de $\mathrm{NaHCO}_{3}$ y $\mathrm{Na}_{2} \mathrm{CO}_{3}(1: 1)$ sin encontrar diferencias significativas con los ensayos con $\mathrm{NaHCO}_{3}$ y $\mathrm{Na}_{2} \mathrm{CO}_{3}$. Conclusión. La solución de $\mathrm{NaHCO}_{3}(50 \mu \mathrm{M})$ logró revertir la apertura bucal suficiente para la toma de evidencia entre las 5- 5,5 horas.
\end{abstract}

Palabras clave: apertura bucal, músculos masticatorios, reversibilidad cadavérica, rigor mortis.

\begin{abstract}
Mandibular reversibility of the cadaverous stiffness by chemical means in a rat model. The main problem that a dentist faces when collecting postmortem evidence is the buccal opening, which is limited by the cadaverous stiffness phenomenon that begins 3 hours after death. This phenomenon persists until it is destroyed by the late autolytic processes of decomposition, after 36 hours postmortem. Objective. To analyze the feasibility of accelerating the reversion of the mandibular cadaverous stiffness by means of chemical substances to facilitate the buccal opening. Materials and methods. We assessed substances capable of altering the $\mathrm{pH}$, chelating intramuscular calcium or inducing proteolysis of the actin-myosin complex of the masticatory muscles with cadaverous stiffness in Wistar rats. Results. We found that mandibular cadaverous stiffness in Wistar rats appears after 2.5 hours of death. After 3.5 hours, once the rigidity was established, we carried out infiltrations with EDTA $(20 \mathrm{mM}), \mathrm{NaHCO}_{3}(50 \mu \mathrm{M}), \mathrm{Na}_{2} \mathrm{CO}_{3}(50 \mu \mathrm{M})$ and papain $(10 \mu \mathrm{M})$. $\mathrm{NaHCO}_{3}$ and $\mathrm{Na}_{2} \mathrm{CO}_{3}$ solutions significantly increased $(p<0.05)$ the reversion speed $(\mathrm{mm} / \mathrm{h})$ from hour 5 , in $108 \%$ and $100 \%$, respectively. Based on the results of this assay, we doubled the concentration of $\mathrm{NaHCO}_{3}$ and assessed a 1:1 mixture of $\mathrm{NaHCO}_{3}$ and $\mathrm{Na}_{2} \mathrm{CO}_{3}$ without finding significant differences with the $\mathrm{NaHCO}_{3}$ and $\mathrm{Na}_{2} \mathrm{CO}_{3}$ prior assays. Conclusion. $\mathrm{NaHCO}_{3}$ solution $(50 \mu \mathrm{M})$ allows a reversal of buccal opening enough to collect evidence between 5 and 5.5 hours.
\end{abstract}

Key words: buccal opening, cadaverous stiffness reversibility, masticatory muscles, rigor mortis. 


\section{Resumo}

Reversibilidade mandibular da rigidez cadavérica por meios químicos num modelo de rato. O principal problema que tem o dentista na toma de evidencias post-mortem é a apertura bucal, limitada pelo fenômeno de rigidez cadavérica, o qual se inicia às 3 horas depois da morte; este fenômeno persiste hasta ser destruído pelos processos autolíticos tardios de decomposição, a partir das 36 horas post-mortem. Objetivo. Estudou-se a possibilidade de acelerar a reversão da rigidez cadavérica mandibular através de substâncias químicas para facilitar a apertura bucal. Materiais e métodos. Foram empregadas substâncias que produzem alteração do pH, quelação do cálcio intramuscular ou proteólises do complexo actina-miosina dos músculos mastigatórios que apresentam rigidez cadavérica em ratos Wistar. Resultados. Estimou-se o tempo de estabelecimento da rigidez cadavérica mandibular no modelo de rato, em 2,5 horas. Às 3,5 horas, logo de estabelecida a rigidez, realizaram-se infiltrações com EDTA $(20 \mathrm{mM}), \mathrm{NaHCO}_{3}(50 \mu \mathrm{M}), \mathrm{Na}_{2} \mathrm{CO}_{3}(50 \mu \mathrm{M})$ e papaína $(10 \mu \mathrm{M})$, encontrando que as soluções de $\mathrm{NaHCO}_{3}$ e $\mathrm{Na}_{2} \mathrm{CO}_{3}$ incrementaram significativamente $(\mathrm{p}<0,05)$ a velocidade de reversão $(\mathrm{mm} / \mathrm{h})$ desde as $5 \mathrm{~h}$, num $108 \%$ e um $100 \%$, respectivamente. A partir deste ensaio duplicou-se a concentração de $\mathrm{NaHCO}_{3}$ e preparou-se uma mistura de $\mathrm{NaHCO}_{3}$ e $\mathrm{Na}_{2} \mathrm{CO}_{3}(1: 1)$ sem obter diferenças significativas com os ensaios com $\mathrm{NaHCO}_{3}$ e $\mathrm{Na}_{2} \mathrm{CO}_{3}$. Conclusão. A solução de $\mathrm{NaHCO}_{3}(50 \mu \mathrm{M})$ reverteu a apertura bucal suficientemente para a toma de evidencias entre as 5- 5,5 horas.

Palavras chave: apertura bucal, músculos mastigatórios, reversibilidade cadavérica, rigor mortis.

\section{Introducción}

En las ciencias forenses es necesario utilizar medios cognoscitivos en la indagación e investigación como elementos materiales probatorios, evidencia física e información; siendo fundamental para el odontólogo forense el manejo apropiado de estos medios y la realización de la prueba pericial en donde realiza una inspección y descripción de la cavidad oral como método de identificación. Esta identificación se puede obtener por medio de cotejos o comparaciones de dos grupos de datos dentales, uno realizado antes de la muerte (antemortem) de la persona desaparecida y los datos obtenidos del cadáver después de la muerte (post mortem) $(1,2,3)$.

El principal problema que recae sobre el odontólogo en la toma de evidencias post mortem es la apertura bucal, limitada generalmente, por los fenómenos tempranos de rigidez cadavérica (rigor mortis) definida como «el estado de dureza, de contracción y de tiesura que sobreviene en los músculos después de la muerte» (4) o como «un estado de contractura que se produce varias horas después de la muerte en todos los músculos del cuerpo» (5). Por otro lado, también se describe como «el estado que se presenta después de la muerte cuando las fibras musculares están completamente depletadas de ATP y de fosforilcreatina, desarrollando un estado de rigidez» (6).

La rigidez en humanos se divide en fases, la primera es la «instauración» que comprende desde su inicio hasta alcanzar su máxima intensidad entre 3 y 24 horas post mortem; en esta fase la rigidez se puede revertir aplicando fuerza y recuperando la flacidez de los miembros, pero después de cierto tiempo, se reinicia este proceso, apareciendo de nuevo la rigidez. En la segunda fase llamada de «estado», de las 24 a las 36 horas, la rigidez es casi irrever- sible y la única forma de hacer que ésta se rompa es produciendo desgarros o fracturas. La tercera y última fase corresponde a la «resolución» de la rigidez que va a partir de las 36 a las 48 horas post mortem, después de esta fase la masa muscular no retorna al rigor; de esta forma en humanos la rigidez cadavérica se evidencia en un periodo de 8 a 12 horas, alcanzando su máxima intensidad a las 24 horas (4). Se han realizado numerosos experimentos para estudiar el desarrollo del rigor mortis en ratas (7-10), tomando intervalos de tiempo de 8 horas, mostrando ascensos en la tensión y endurecimiento de los músculos de las 2 a las 4 horas aproximadamente, con temperaturas de $25^{\circ} \mathrm{C}$ a $37^{\circ} \mathrm{C}$. También en el modelo de rata se ha observado las tres fases de rigor post mortem, incrementándose la intensidad en un lapso de tiempo menor de 5 horas, presentándose reversión desde las 7 horas y a las 24 horas ya no se presenta rigidez cadavérica (7). La rigidez cadavérica también se ve afectada por la temperatura, los acontecimientos previos a la muerte y la causa de muerte $(4,7,11)$.

Inmediatamente después de la muerte, se produce normalmente un estado de relajación y flacidez de todos los músculos del cuerpo, al cabo de un cierto tiempo se inicia el proceso de contractura muscular o rigidez cadavérica, ésta se debe a una reacción del tejido muscular que se hace ácido, siendo proporcional el aumento de la rigidez al aumento de acidez. Por tanto, la rigidez cadavérica no comienza sino hasta cuando se agotan las reservas de energía del músculo, dependiente del glucógeno existente; en donde en la etapa post mortem el glucógeno se convierte en ácido láctico en anaerobiosis, por la glicólisis post mortem $(4,12)$. Se ha demostrado que los cambios biológicos post mortem y el rigor mortis no progresan simultáneamente en todos los músculos (11), además el volumen muscular no afecta ni el progreso, ni la resolución el rigor (9). 
Cuando se trata de lograr movimiento de las articulaciones para examinar la rigidez, es necesario tener en cuenta que algunas articulaciones, como la articulación temporomandibular, no son fáciles de mover (9). La elevación de la mandíbula hacia el hueso maxilar y sus estructuras dentarias, está controlada por la acción de los músculos masticatorios donde se resaltan el masetero y el temporal encargados del cierre de la cavidad oral. Después de la muerte, los niveles de ATP empiezan a descender a la hora post mortem en los músculos masetero y temporal por ausencia de oxígeno $(10,11)$.

Existen diversas técnicas para lograr la apertura mandibular, las cuales son agresivas y producen daños faciales estéticos que afectan psicológicamente la familia y por lo cual muchas veces reclaman; o simplemente no se permite ver el cadáver, por los daños producidos a las estructuras anatómicas, originados por excesivas fuerzas mecánicas, recesiones maxilares o miotomías $(3,13,14)$.

Después de analizar todos los procesos bioquímicos, biológicos y circunstancias que influyen sobre la rigidez cadavérica, se planteó adelantar el proceso de reversión de la rigidez para facilitar el examen intraoral, aplicando sustancias que podrían acelerar la reversión mandibular en el modelo de rata.

\section{Materiales y métodos}

Se trabajó con ratas Wistar adultas (mayores de 2 meses). Los animales, durante su crecimiento, fueron alimentados ad libitum y mantenidos a una temperatura entre los 25$30^{\circ} \mathrm{C}$, con una humedad de $45-55 \%$. El sacrificio se realizó por dislocación cervical, registrándose inmediatamente el valor de apertura bucal máxima $(\mathrm{mm})$ con un calibrador pie de rey. Las medidas de apertura bucal se tomaron entre los bordes incisales de los incisivos superiores e inferiores, midiendo en milímetros el espacio comprendido entre éstos, en todos los grupos de ratas. Estas mediciones se realizaron a $25-27^{\circ} \mathrm{C} ; 45-52 \%$ de humedad relativa, a diferentes tiempos post mortem desde las 0 a 8 horas tomando intervalos de 30 minutos. Adicionalmente, una vez tomada la apertura en el tiempo inicial, se pesaron los animales.

\section{Primer ensayo}

Se emplearon sustancias que por infiltración en los músculos masticadores favorecieran la variación en el $\mathrm{pH}$, la variación de la concentración de calcio disponible (15) o la adición de una enzima proteolítica (16).
De acuerdo a la literatura estudiada, la acidificación de los músculos es uno de los principales cambios que ocurren después de la muerte; la variación en el grado y la extensión de la acidificación influyen en la capacidad de retención del agua. Las proteínas miofibrilares, alcanzan un $\mathrm{pH}$ en el cual las moléculas proteicas no tienen carga eléctrica neta (punto isoeléctrico) y tienden a perder el agua que está normalmente unida a ellas, generando una exudación de fluidos de las fibras musculares. La concentración de glucógeno se convierte en el limitante para que se dé el $\mathrm{pH}$ final que es inversamente proporcional a la concentración de lactato (19). Para determinar la concentración de las sales $\mathrm{NaHCO}_{3}$ y $\mathrm{Na}_{2} \mathrm{CO}_{3}$ se tuvo en cuenta los niveles de ácido láctico $(50 \mu \mathrm{M})$ producido en condiciones anaeróbicas en los músculos masticadores en ratas a las 2 horas post mortem (10), buscando neutralizar el ácido láctico.

Para la disminución de la concentración de calcio disponible se debe tener en cuenta que dentro de las proteínas musculares se encuentra la troponina $\mathrm{C}$, por cada una de estas proteínas, se fijan 4 moléculas de $\mathrm{Ca}^{+2}$. Conjuntamente la afinidad de la troponina $\mathrm{C}$ por el calcio disminuye en condiciones ácidas (20). La elección de la concentración de EDTA, se basó en experimentos realizados con el fin de reducir las cadenas livianas de miosina y de troponina $\mathrm{C}$, sumergiendo segmentos de fibra muscular en una solución de $20 \mathrm{mM}$; el tratamiento con EDTA resultó en una reducción sustancial de la tensión, debido principalmente a la pérdida de la troponina $\mathrm{C}$ y en pequeñas reducciones, por la extracción de las cadenas livianas de miosina (15).

Una de las razones por las que el músculo se contrae post mortem corresponde a la pérdida de la capacidad para secuestrar calcio del retículo sarcoplásmico y de las mitocondrias, y se debe probablemente, al deterioro de los sistemas que generan energía al músculo y al aumento de la permeabilidad que estos organelos exhiben a lo largo del tiempo post mortem bajo condiciones anóxicas (permitiendo una mayor pérdida del calcio de las membranas) (20). Se requieren 2 moléculas de EDTA por 3 calcios, este quelato ha sido utilizado en estudios previos, en donde a animales vivos, se les realizaron inyecciones intravenosas de EDTA, previniendo la inextensibilidad que normalmente ocurre durante las 7 horas post mortem, concluyendo que un cierto nivel de calcio libre es requerido para el desarrollo del rigor mortis (21). Por lo tanto, se tomó la concentración EDTA ( $20 \mathrm{mM})$, necesaria para reducir las cadenas livianas de miosina y de troponina $\mathrm{C}$ al acomplejar el calcio libre. 
Adicionalmente se pensó que la adición de una enzima proteolítica pudiera favorecer la reversibilidad, se eligió la papaína (EC 3.4.22.2) $(10 \mu \mathrm{M})$ con base en su acción proteolítica que puede producir digestión de la miosina ATPasa (EC 3.6.4.1) y con base a que la máxima actividad de esta enzima es a $\mathrm{pH}$ ácido $(\mathrm{pH}=5), \mathrm{pH}$ que se encuentra post mortem en el modelo de rata. Por otro lado, la molécula de miosina está compuesta por un bastón, el cual está subdividido en dos partes denominadas meromiosina ligera (LMM) y pesada (HMM), en uno de los extremos la cadena pesada se pliega formando las cabezas globulares SF1 ó S1; estas cabezas globulares son las que se unen a la actina en la contracción. Según estudios de proteólisis controlada para determinar la disposición de las subunidades en la molécula, los puntos de unión de la meromiosina ligera y de la cabeza de la molécula de miosina, son susceptibles al ataque por las enzimas proteolíticas. La papaína corta la cadena pesada de la miosina de las cabezas globulares SF1 ó S1 $(22,23)$.

En resumen, se aplicaron para las infiltraciones en el primer ensayo el bicarbonato de sodio $\left(\mathrm{NaHCO}_{3}\right)(50 \mu \mathrm{M})(\mathrm{pH}$ de 8.39 a $\left.21^{\circ} \mathrm{C}\right)$ y el carbonato de sodio $\left(\mathrm{Na}_{2} \mathrm{CO}_{3}\right)(50 \mu \mathrm{M})$ $\left(\mathrm{pH}\right.$ de 11,25 a $\left.21^{\circ} \mathrm{C}\right)(10)$, EDTA disódico $(20 \mathrm{mM}) \mathrm{y}$ papaína $(10 \mu \mathrm{M})$.

\section{Infiltraciones}

Se realizaron en un momento de la fase estado donde se mantiene la rigidez cadavérica; en los siguientes puntos anatómicos, mediante una línea imaginaria desde el tragus hasta el canto externo del ojo y en dirección a esta línea a $5 \mathrm{~mm}$ del tragus aproximadamente. El segundo punto se infiltró mediante la palpación del ángulo de la mandíbula, trazando una línea perpendicular hasta la parte media del arco zigomático y sobre el punto medio de esta perpendicular se aplica la sustancia, utilizando jeringas de $1 \mathrm{ml}$. Este ensayo incluyó: un grupo testigo, que consistió en animales a los que no se le hizo ningún tipo de infiltración; un grupo control, con la aplicación infiltrativa del vehículo (agua desionizada) y los grupos con las diferentes sustancias estudiadas, infiltrados en los puntos anatómicos descritos anteriormente.

\section{Análisis estadístico}

Se realizó un análisis de varianza (ANOVA) entre cada uno de los tratamientos a los diferentes tiempos post mortem, para determinar los efectos de los principales factores considerados. Se realizó un análisis de regresión simple tomando las aperturas, como variables dependientes y el tiempo como variable independiente. De acuerdo a los resultados, se seleccionaron los dos grupos de sustancias más rápidos y eficaces, logrando conformar dos grupos de mezclas para el segundo ensayo.

\section{Segundo ensayo}

Se duplicó la concentración de $\mathrm{NaHCO}_{3}(100 \mu \mathrm{M})(\mathrm{pH}$ de $8,18)$ y se creó una mezcla $1: 1$ de dos soluciones con $\mathrm{NaHCO}_{3}(50 \mu \mathrm{M})$ y carbonato de sodio $\mathrm{Na}_{2} \mathrm{CO}_{3}(50 \mu \mathrm{M})(\mathrm{pH}$ de 10). Los resultados de apertura se compararon estadísticamente con los resultados del testigo y el control. Se realizó un análisis de varianza (ANOVA) y se aplicó la prueba de comparación múltiple de Duncan. Se tomó como criterio de efectividad de la reversibilidad cadavérica para la toma de evidencia bucal cuando la apertura alcance el $50 \%$ en el menor tiempo posible.

Como un indicador de la velocidad de reversión de la rigidez se tomaron los valores a partir de las 5 horas hasta las 8 horas, donde se empezaron a observar diferencias significativas $(\mathrm{p}<0,05)$. Se calcularon los valores de las curvas de tendencia lineal, cuyo coeficiente de correlación lineal fuera cercano a 1. Las pendientes de estas curvas se utilizaron arbitrariamente como indicadores de la reversión.

\section{Resultados y discusión}

\section{Determinación de la rigidez cadavérica}

El análisis estadístico indica que la fase de instauración de la rigidez se propagó durante 2.5 horas $(150 \mathrm{~min})$ presentándose una caída significativa $(\mathrm{p}<0,05)$ desde los $60 \mathrm{mi}-$ nutos hasta los 150 minutos (Tabla 1, Figura 1). Entre las 3 horas (180 min) hasta las 7 horas (420 min) se entró en la fase de estado manteniéndose estadísticamente constante; a partir de las 7,5 horas (420 min) inició la fase de resolución, donde la apertura bucal alcanzó valores de 59\%, llegando a valores de $62 \%$ de apertura a las 8 horas. Esta reversión se puede atribuir a la acción de las enzimas lisosomales liberadas en la autolisis post mortem, que destruyen las proteínas musculares, terminando con esto la rigidez cadavérica perteneciente a los fenómenos cadavéricos tempranos y dando inicio a los tardíos como la descomposición; favorecida por microorganismos que pueden actuar sobre los componentes orgánicos desprotegidos (23). Este valor coincide con estudios del desarrollo de la intensidad del rigor mortis en ratas albinas macho del mismo peso y la misma edad, encontrando que a partir de las 7 horas a $24.5^{\circ} \mathrm{C}$ se empieza a disminuir la intensidad de la rigidez en los músculos de miembros inferiores, 
Tabla 1. Desarrollo de la rigidez cadavérica. La apertura bucal máxima se tomó con un calibrador pie de rey entre los bordes incisales de los incisivos superiores e inferiores, midiendo en milímetros $(\mathrm{mm})$ el espacio comprendido entre éstos. Las mediciones se realizaron a $25-27^{\circ} \mathrm{C} ; 45-52 \%$ de humedad relativa, a diferentes tiempos post mortem desde las 0 a 8 horas tomando intervalos de 30 minutos. Se presentan los valores promedio \pm $\operatorname{SEM}(n=6)$. Adicionalmente el delta de apertura $(\Delta)$ y el porcentaje de apertura bucal.

\begin{tabular}{cccc}
\hline Tiempo $(\mathbf{h})$ & Apertura $(\mathbf{m m})$ & $\boldsymbol{\Delta}$ apertura & \% apertura \\
\hline 0,0 & $23,3 \pm 2,0$ & & 100 \\
0,5 & $21,2 \pm 1,3$ & $-2,1^{*}$ & 91 \\
1,0 & $20,0 \pm 0,9$ & $-1,2$ & 86 \\
1,5 & $17,7 \pm 2,2$ & $-2,3^{*}$ & 76 \\
2,0 & $12,5 \pm 2,1$ & $-5,2^{*}$ & 54 \\
2,5 & $7,8 \pm 1,8$ & $-4,7^{*}$ & 34 \\
3,0 & $7,0 \pm 1,7$ & $-0,8$ & 30 \\
3,5 & $7,0 \pm 1,7$ & 0 & 30 \\
4,0 & $6,8 \pm 1,5$ & $-0,2$ & 29 \\
4,5 & $6,8 \pm 1,7$ & 0 & 29 \\
5,0 & $7,3 \pm 1,5$ & 0,5 & 31 \\
5,5 & $8,2 \pm 1,3$ & 0,8 & 35 \\
6,0 & $8,5 \pm 2,2$ & 0,3 & 44 \\
6,5 & $10,2 \pm 3,1$ & 1,7 & 47 \\
7,0 & $11,0 \pm 3,3$ & 0,8 & 59 \\
7,5 & $13,7 \pm 3,4$ & 2,7 & 62 \\
8,0 & $14,5 \pm 3,3$ & 0,8 & \\
\hline
\end{tabular}

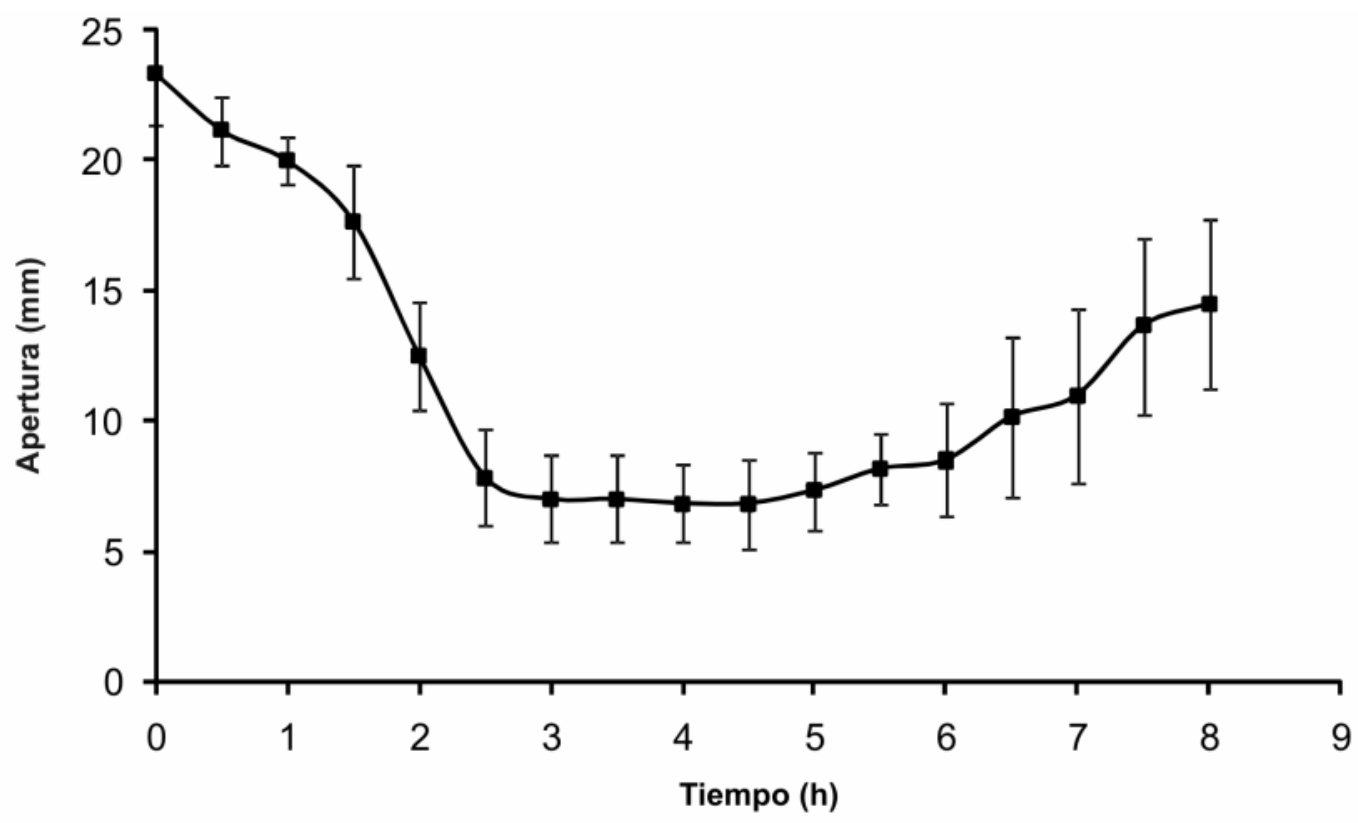

Figura 1. Desarrollo de la rigidez cadavérica en el grupo testigo sin vehículo (agua). El valor de apertura bucal máxima se tomó con un calibrador pie de rey entre los bordes incisales de los incisivos superiores e inferiores, midiendo en milímetros ( $\mathrm{mm}$ ) el espacio comprendido entre éstos. Las mediciones se realizaron a $25-27^{\circ} \mathrm{C} ; 45-52 \%$ de humedad relativa, a diferentes tiempos post mortem desde las 0 a 8 horas tomando intervalos de 30 minutos. Se presentan los valores promedio \pm SEM ( $\mathrm{n}=6)$. 
estos músculos poseen fibras tipo I y tipo IIA, que son fibras similares a las encontradas en el músculo masetero y fibras tipo IIB encontradas en el músculo temporal (11); las fibras tipo I muestran una distribución y un patrón de tinción desde el perfil histoenzimológico comparable al tipo de fibra de los otros músculos esqueléticos en su uniformidad del tamaño de fibra; y su diámetro dobla el número de fibras tipo II (17), dándoles a estos músculos masticadores, características únicas de músculos rojos, siendo de esta forma más rápida la contracción en este tipo de fibras debido al metabolismo de fosforilación oxidativa para la formación de ATP, el cual cae rápidamente por la ausencia de oxígeno $(11,18)$.

Por otro lado, las ratas utilizadas en este ensayo (39 ratas) eran machos y hembras cuyos pesos variaron entre 313,7 $\pm 43,0$ g. Los animales se agruparon en 5 grupos: 150200; 200-250; 250-300: 300-350 y de 350-400 g, para determinar si la variación en peso, tenía un efecto sobre la instauración de la rigidez. Los resultados estadísticos no evidenciaron diferencias significativas $(\mathrm{p}<0,05)$ entre los diferentes grupos y la instauración de la rigidez ocurrió en el mismo lapso de tiempo en todos los animales. Por lo tanto, el peso de los animales no afectó la instauración de la rigidez. Estos resultados coinciden con los resultados obtenidos en ratas de diferentes pesos, donde el tiempo de curso de desarrollo de la rigidez fue siempre el mismo (7).

De acuerdo a estos resultados, se esperó que se estabilizara la fase de estado, cuando el delta de apertura ( $\ddot{A})$ fue igual a cero, que fue a las 3,5 horas (Tabla 1). Tomándose este tiempo como el tiempo inicial para realizar las intervenciones y se tomó el valor de 7,5 horas como el valor a disminuir con las intervenciones.

\section{Ensayos de reversión}

Se emplearon sustancias que por infiltración en los músculos masticadores, favorecieran la variación en el pH, la variación de la concentración de calcio disponible o la hidrólisis de proteínas, se utilizó como vehículo agua desionizada que sirvió como control del ensayo. Los resultados de la comparación de sustancias infiltradas en el primer ensayo donde se aplicaron a las $3.5 \mathrm{~h}$ post mortem infiltraciones en los músculos masticatorios de bicarbonato de sodio $\left(\mathrm{NaHCO}_{3}\right)(50 \mu \mathrm{M})\left(\mathrm{pH}\right.$ de 8,39 a $\left.21^{\circ} \mathrm{C}\right)$; el carbonato de sodio $\left(\mathrm{Na}_{2} \mathrm{CO}_{3}\right)(50 \mu \mathrm{M})\left(\mathrm{pH}\right.$ de 11,25 a $\left.21^{\circ} \mathrm{C}\right)$; EDTA disódico $(20 \mathrm{mM})$ y papaína $(10 \mu \mathrm{M})$ aparecen en la
Tabla 2 y Figura 2. Las pendientes de las curvas que se utilizaron como indicadores de velocidad aparecen en la Tabla 3.

\section{Variación del control}

La inyección del vehículo fue similar al testigo entre las 4 y 5,5 horas, no encontrándose variaciones en la apertura; sin embargo, a partir de las 6 horas la hidratación del tejido empezó a favorecer la reversión alcanzando un $42 \%$ de la apertura bucal inicial, y a las 8 horas los valores fueron de $73 \%$. Los resultados indican que la hidratación de los músculos masticatorios presentaron una reversibilidad suficiente para la toma de evidencia entre las 6,5 y 7 horas (Tabla 2). Por otro lado, el indicador de velocidad señala que la hidratación incrementó un 30\% la velocidad de reversión de la rigidez (Tabla 3).

\section{Variación en el pH}

La inyección de la solución de $\mathrm{Na}_{2} \mathrm{CO}_{3}$ fue similar al testigo y al control entre las 4 y 5,5 horas, no encontrándose variaciones en la apertura; sin embargo, a partir de las 6 horas la neutralización de la acidez en el tejido empezó a favorecer la reversión alcanzando un 55\% de la apertura bucal inicial y a las 8 horas los valores fueron de $95 \%$, mejorando aun los valores obtenidos por la hidratación del tejido. Los resultados indican que la neutralización de la acidez en los músculos masticatorios favoreció la reversibilidad suficiente para la toma de evidencia entre las 5,5 y 6 horas (Tabla 2). Los indicadores de velocidad señalan que la neutralización incrementó un $100 \%$ la velocidad de reversión de la rigidez respecto al tratamiento testigo y un 53\% con respecto al tratamiento control (Tabla 3).

La inyección de la solución de $\mathrm{NaHCO}_{3}$ fue similar al testigo y al control entre las 4 y 4,5 horas, no encontrándose variaciones en la apertura; sin embargo, a partir de las 5 horas la neutralización de la acidez en el tejido empezó a favorecer la reversión alcanzando un $38 \%$ de la apertura bucal inicial y a las 8 horas los valores fueron de 97\%. Los resultados indican que la neutralización de los músculos masticatorios presentaron una reversibilidad suficiente para la toma de evidencia entre las 5 y 5,5 horas (Tabla 2). Los indicadores de velocidad señalan que la neutralización incrementó un $108 \%$ la velocidad de reversión de la rigidez con respecto al tratamiento testigo y un $60 \%$ respecto al tratamiento control (Tabla 3). 
Tabla 2. Comparación de sustancias infiltradas en el primer ensayo. Se aplicaron a las $3.5 \mathrm{~h}$ post mortem infiltraciones en los músculos masticatorios de bicarbonato de sodio $\left(\mathrm{NaHCO}_{3}\right)(50 \mu \mathrm{M})\left(\mathrm{pH} \mathrm{de} \mathrm{8,39} \mathrm{a} 21^{\circ} \mathrm{C}\right)$; el carbonato de sodio $\left(\mathrm{Na}_{2} \mathrm{CO}_{3}\right)(50 \mu \mathrm{M})\left(\mathrm{pH}\right.$ de 11,25 a $\left.21^{\circ} \mathrm{C}\right)$; EDTA disódico $(20 \mathrm{mM})$ y papaína $(10 \mu \mathrm{M})$. Se presentan los valores promedio \pm SEM.

\begin{tabular}{|c|c|c|c|c|c|c|c|c|c|c|c|c|}
\hline \multirow[b]{2}{*}{ Tiempo } & \multicolumn{2}{|c|}{$\begin{array}{l}\text { Testigo } \\
(\mathbf{n}=3)\end{array}$} & \multicolumn{2}{|c|}{$\begin{array}{c}\text { Control } \\
(\mathrm{n}=6)\end{array}$} & \multicolumn{2}{|c|}{$\begin{array}{c}\mathrm{NaHCO}_{3} \\
(50 \mu \mathrm{M}) \\
(\mathbf{n}=\mathbf{3})\end{array}$} & \multicolumn{2}{|l|}{$\begin{array}{c}\mathrm{Na}_{2} \mathrm{CO}_{3} \\
(50 \mu \mathrm{M}) \\
(\mathrm{n}=3)\end{array}$} & \multicolumn{2}{|c|}{$\begin{array}{c}\text { EDTA } \\
(20 \mathrm{mM}) \\
(\mathbf{n}=\mathbf{3})\end{array}$} & \multicolumn{2}{|l|}{$\begin{array}{l}\text { Papaína } \\
(10 \mu M) \\
(\mathbf{n}=3)\end{array}$} \\
\hline & Apertura & $\%$ & Apertura & $\%$ & Apertura & $\%$ & Apertura & $\%$ & Apertura & $\%$ & Apertura & $\%$ \\
\hline $\mathbf{0 , 0}$ & $24,3 \pm 2.3$ & 100 & $22,5 \pm 1,2$ & 100 & $24,7 \pm 1,2$ & 100 & $21,3 \pm 1,5$ & 100 & $21,7 \pm 1,0$ & 100 & $22,0 \pm 1,0$ & 100 \\
\hline 0,5 & $22,0 \pm 1,0$ & 90 & $20,8 \pm 0,6$ & 93 & $21,7 \pm 1,2$ & 88 & $19,0 \pm 0,0$ & 89 & $20,0 \pm 0,6$ & 92 & $20,3 \pm 0,6$ & 92 \\
\hline 1,0 & $20,3 \pm 1,2$ & 84 & $19,2 \pm 1,5$ & 85 & $19,3 \pm 1,2$ & 78 & $16,3 \pm 0,6$ & $\pi$ & $18,0 \pm 0,6$ & 83 & $18,3 \pm 0,6$ & 83 \\
\hline 1,5 & $19,0 \pm 0,0$ & 78 & $16,5 \pm 0,6$ & 73 & $16,0 \pm 1,7$ & 65 & $15,7 \pm 0,6$ & 73 & $15,7 \pm 1,5$ & 72 & $15,7 \pm 1,5$ & 71 \\
\hline 2,0 & $13,0 \pm 1,0$ & 53 & $12,8 \pm 1,7$ & 57 & $8,0 \pm 2,6$ & 32 & $14,0 \pm 1,0$ & 66 & $13,0 \pm 1,2$ & 60 & $12,7 \pm 1,2$ & 58 \\
\hline 2,5 & $7,3 \pm 1,5$ & 30 & $9,2 \pm 0,6$ & 41 & $5,3 \pm 0,6$ & 22 & $9,7 \pm 1,5$ & 45 & $9,7 \pm 2,0$ & 45 & $8,0 \pm 2,0$ & 36 \\
\hline 3,0 & $6,0 \pm 1,7$ & 25 & $7,5 \pm 0,0$ & 33 & $4,7 \pm 0,6$ & 19 & $7,7 \pm 1,2$ & 36 & $7,7 \pm 1,5$ & 35 & $6,7 \pm 1,5$ & 30 \\
\hline 3,5 & $6,0 \pm 1,7$ & 25 & $6,8 \pm 0,0$ & 30 & $4,7 \pm 0,6$ & 19 & $7,7 \pm 1,2$ & 36 & $7,0 \pm 1,7$ & 32 & $6,7 \pm 1,5$ & 30 \\
\hline 4,0 & $6,0 \pm 1,7$ & 25 & $6,7 \pm 0,6$ & 30 & $6,0 \pm 0,0$ & 24 & $7,3 \pm 0,6$ & 34 & $7,0 \pm 1,7$ & 32 & $6,0 \pm 1,7$ & 27 \\
\hline 4,5 & $6,0 \pm 1,7$ & 25 & $7,2 \pm 0,6$ & 32 & $7,3 \pm 0,6$ & 30 & $7,7 \pm 0,6$ & 36 & $7,3 \pm 2,1$ & 34 & $7,0 \pm 1,7$ & 32 \\
\hline 5,0 & $6,3 \pm 1,5$ & 26 & $7,7 \pm 0,6$ & 34 & $9,3 \pm 1,5$ & 38 & $7,7 \pm 0,6$ & 36 & $7,3 \pm 2,1$ & 34 & $7,0 \pm 1,7$ & 32 \\
\hline 5,5 & $7,3 \pm 1,5$ & 30 & $8,3 \pm 1,0$ & 37 & $13,7 \pm 1,5$ & 55 & $8,3 \pm 0,6$ & 39 & $8,0 \pm 1,7$ & 37 & $7,3 \pm 2,1$ & 33 \\
\hline 6,0 & $7,0 \pm 2,0$ & 29 & $9,5 \pm 2,5$ & 42 & $17,3 \pm 2,3$ & 70 & $11,7 \pm 1,2$ & 55 & $10,0 \pm 2,6$ & 46 & $11,0 \pm 1,7$ & 50 \\
\hline 6,5 & $8,0 \pm 2,0$ & 33 & $11,0 \pm 3,1$ & 49 & $20,3 \pm 1,5$ & 82 & $14,0 \pm 1,0$ & 66 & $12,3 \pm 1,5$ & 57 & $13,7 \pm 2,5$ & 62 \\
\hline 7,0 & $8,7 \pm 2,5$ & 36 & $13,7 \pm 3,5$ & 61 & $21,3 \pm 1,2$ & 86 & $17,0 \pm 1,0$ & 80 & $15,0 \pm 1,7$ & 69 & $15,7 \pm 2,3$ & 71 \\
\hline 7,5 & $11,7 \pm 3,5$ & 48 & $14,3 \pm 4,0$ & 64 & $23,3 \pm 1,2$ & 95 & $19,0 \pm 1,0$ & 89 & $15,0 \pm 1,7$ & 69 & $17,0 \pm 2,6$ & $\pi$ \\
\hline 8,0 & $13,7 \pm 4,7$ & 56 & $16,3 \pm 5,5$ & 73 & $24,0 \pm 0,0$ & 97 & $20,3 \pm 1,2$ & 95 & $16,0 \pm 2,0$ & 74 & $19,0 \pm 3,6$ & 86 \\
\hline
\end{tabular}




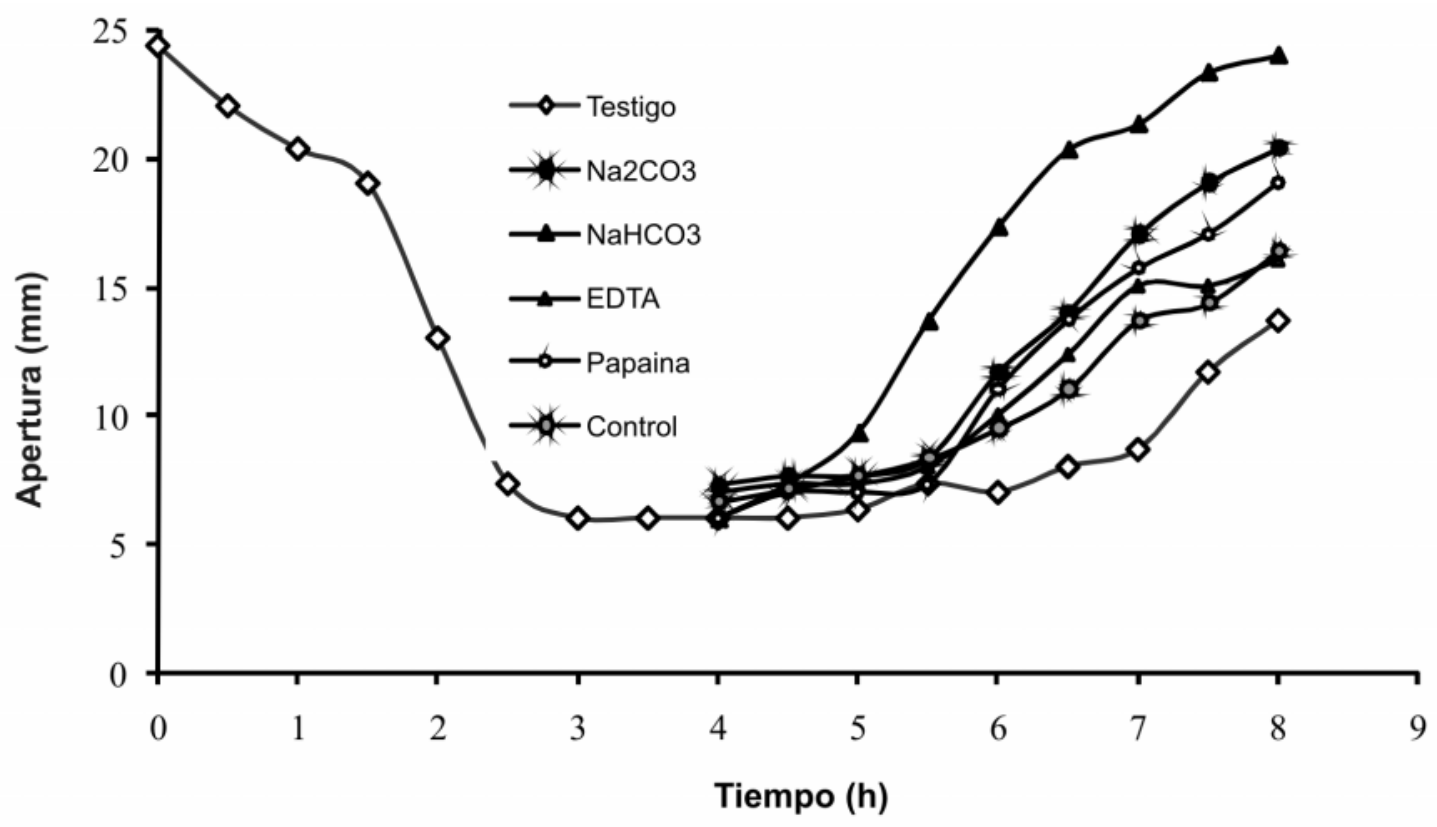

Figura 2. Comparación de sustancias en el primer ensayo. Se aplicaron a las $3,5 \mathrm{~h}$ post mortem infiltraciones en los músculos masticatorios de bicarbonato de sodio $\left(\mathrm{NaHCO}_{3}\right)(50 \mu \mathrm{M})\left(\mathrm{pH}\right.$ de 8,39 a $\left.21^{\circ} \mathrm{C}\right)$; el carbonato de sodio $(\mathrm{Na} \mathrm{CO})(50$ $\mu \mathrm{M})\left(\mathrm{pH}\right.$ de $\left.11,25 \mathrm{a} 21^{\circ} \mathrm{C}\right)$; EDTA disódico $(20 \mathrm{mM})$ y papaína $(10 \mu \mathrm{M})$. Los resultados desde las $4 \mathrm{~h}$ se comparân cỏn el tratamiento testigo ( in vehículo) y con el tratamiento control (con vehículo). Se presentan los valores promedio \pm SEM.

Tabla 3. Velocidad de las sustancias. Como un indicador de la velocidad de reversión de la rigidez en el primer ensayo se tomaron los valores a partir de las 5 horas hasta las 8 horas, donde se empezaron a observar diferencias significativas $(\mathbf{p}<0,05)$. Se calcularon los valores de las curvas de tendencia lineal, cuyo coeficiente de correlación lineal $\left(r^{2}\right)$ fuera cercano a 1 . Las pendientes de estas curvas se utilizaron arbitrariamente como indicadores de la velocidad de reversión cadavérica mandibular.

\begin{tabular}{lccc}
\hline & Pendiente $(\mathbf{m m} / \mathbf{h})$ & Intercepto $(\mathbf{m m})$ & $\mathbf{r}^{\mathbf{2}}$ \\
\hline Testigo & 2,3095 & 6,0595 & 0,8510 \\
Control & 3,0119 & 8,0298 & 0,9763 \\
$\mathrm{NaHCO}_{3}(50 \mu \mathrm{M})$ & 4,8095 & 12,7860 & 0,9319 \\
$\mathrm{Na}_{2} \mathrm{CO}_{3}(50 \mu \mathrm{M})$ & 4,6190 & 16,0240 & 0,9840 \\
EDTA $(20 \mu \mathrm{M})$ & 3,2143 & 8,9405 & 0,9532 \\
Papaína $(10 \mu \mathrm{M})$ & 4,2857 & 14,9050 & 0,9766 \\
\hline
\end{tabular}




\section{Variación de la concentración de calcio disponible}

La inyección de la solución de EDTA fue similar al testigo y al control entre las 4 y 5,5 horas, no encontrándose variaciones en la apertura; sin embargo, a partir de las 6 horas el acomplejamiento del calcio libre en el tejido empezó a favorecer la reversión alcanzando un $46 \%$ de la apertura bucal inicial y a las 8 horas los valores fueron de $74 \%$. Los resultados indican que la neutralización del calcio de los músculos masticatorios presentaron una reversibilidad suficiente para la toma de evidencia entre las 6 y 6,5 horas (Tabla 2). Los indicadores de velocidad señalan que la neutralización de calcio incrementó 39\% la velocidad de reversión de la rigidez con respecto al tratamiento testigo y un $7 \%$ respecto al tratamiento control (Tabla 3).

\section{Hidrólisis de las proteínas}

La inyección de la solución de papaína fue similar al testigo y al control entre las 4 y 5,5 horas, no encontrándose variaciones en la apertura; sin embargo, a partir de las 6 horas la proteólisis en el tejido empezó a favorecer la reversión alcanzando un 50\% de la apertura bucal inicial y a las 8 horas los valores fueron de $86 \%$. Los resultados indican que la proteólisis en los músculos masticatorios presentaron una reversibilidad suficiente para la toma de evidencia a las 6 horas (Tabla 2). Los indicadores de velocidad señalan que la proteólisis incrementó un $86 \%$ la velocidad de reversión de la rigidez con respecto al tratamiento testigo y un $42 \%$ respecto al tratamiento control (Tabla 3).

Se encontró que las soluciones de $\mathrm{NaHCO}_{3}$ y $\mathrm{Na}_{2} \mathrm{CO}_{3}$, fueron las que mostraron mayor reversibilidad, seguidos por la papaína; el EDTA presentó un comportamiento similar al control; el control no presentó diferencias significativas con el grupo testigo; se alcanzó a observar el efecto del agua desionizada (vehículo) con respecto al grupo testigo pero ésta no fue significativa. Con respecto a la papaína se observó reversibilidad similar al carbonato, no obstante, no se hizo ensayos con una mayor concentración de esta proteína, ya que puede incrementar los costos de la prueba.

Las pendientes de las curvas se utilizaron como indicadores de velocidad aparecen en la Tabla 3. De acuerdo a la velocidad de reversibilidad demostrada a partir de las 4:30:00 horas, los valores de la pendiente despejando la ecuación en donde $\mathrm{Y}$ es apertura y $\mathrm{X}$ tiem- po; se confirmó que las 2 mejores sustancias que mostraron un comportamiento más veloz fueron el $\mathrm{NaHCO} 3$ y $\mathrm{Na} 2 \mathrm{CO} 3$ respectivamente. De acuerdo a estos criterios se escogieron estas sustancias para realizar el ensayo final que buscaría acelerar aun más la velocidad de reversión.

\section{Ensayo final}

Los resultados previos demostraron que la variación de $\mathrm{pH}$ fue la mejor opción para revertir la rigidez. Se varió la concentración bicarbonato para un $\mathrm{pH}$ de $8,18 \mathrm{y}$ una mezcla de $\mathrm{NaHCO}_{3}$ y $\mathrm{Na}_{2} \mathrm{CO}_{3}(1: 1)$ para $\mathrm{pH} 10$, para ver si se mejoraba aun más la reversibilidad encontrada en el experimento anterior.

La inyección de la solución doble de $\mathrm{NaHCO}_{3}$ fue similar al testigo y al control entre las 0 y 5 horas, no encontrándose variaciones en la apertura, no obstante, a partir de las 5,5 horas la neutralización de la acidez en el tejido empezó a favorecer la reversión alcanzando un $49 \%$ $(\mathrm{p}<0,05)$ de la apertura bucal inicial y a las 8 horas los valores fueron de $94 \%(p<0,05)$. Los resultados indican que la neutralización de la acidez de los músculos masticatorios causados por el bicarbonato presentaron una reversibilidad suficiente para la toma de evidencia entre las 5,5 y 6 horas (Tabla 4, Figura 3). Los indicadores de velocidad señalan que la neutralización incrementó un $88 \%$ la velocidad de reversión de la rigidez con respecto al tratamiento testigo y un $56 \%$ respecto al tratamiento control (Tabla 5).

La inyección de la solución de $\mathrm{NaHCO}_{3}$ y $\mathrm{Na}_{2} \mathrm{CO}_{3}(1: 1)$ fue similar estadísticamente $(\mathrm{p}<0.05)$ al testigo y al control entre las 0 y 4,5 horas, no encontrándose variaciones en la apertura; sin embargo, a partir de las 5 horas la neutralización de la acidez en el tejido empezó a favorecer la reversión alcanzando un $46 \%(\mathrm{p}<0,05)$ de la apertura bucal inicial y a las 8 horas los valores fueron de $97 \%$ ( $p<0,05)$. Los resultados indican que la neutralización de la acidez de los músculos masticatorios causados por la mezcla presentaron una reversibilidad suficiente para la toma de evidencia entre las 5 y 5,5 horas (Tabla 4). Los indicadores de velocidad señalan que la neutralización incrementó un 58\% la velocidad de reversión de la rigidez con respecto al tratamiento testigo y un $31 \%$ respecto al tratamiento control (Tabla 5).

Con base a la velocidad de la reversibilidad podríamos predecir a cuántas horas se produciría una reversión de la rigidez a partir de las 5:00:00 horas, para tener una apertura igual a la 
Tabla 4. Comparación de sustancias infiltradas en el segundo ensayo. Se aplicaron a las $3.5 \mathrm{~h}$ post mortem infiltraciones en los músculos masticatorios de bicarbonato de sodio $\mathrm{NaHCO}_{3}(100 \mu \mathrm{M})(\mathrm{pH}$ de 8,18) y se creó una mezcla 1:1 de dos soluciones con $\mathrm{NaHCO}_{3}(50 \mu \mathrm{M})$ y carbonato de sodio $\mathrm{Na}_{2} \mathrm{CO}_{3}(50 \mu \mathrm{M})(\mathrm{pH}$ de 10). Los resultados a partir de las $3.5 \mathrm{~h}$ se comparan estadísticamente con el tratamiento testigo (sin vehículo) y con el tratamiento control (con vehículo). Se presentan los valores promedio \pm SEM. Las diferencias significativas en cada tiempo se expresan con letras mayúsculas.

\begin{tabular}{|c|c|c|c|c|c|c|c|c|c|c|c|c|}
\hline \multirow[b]{2}{*}{ Tiempo } & \multicolumn{3}{|c|}{$\begin{array}{l}\text { Testigo } \\
(\mathbf{n}=\mathbf{6})\end{array}$} & \multicolumn{3}{|c|}{$\begin{array}{l}\text { Control } \\
(\mathbf{n}=6)\end{array}$} & \multicolumn{3}{|c|}{$\begin{array}{c}\mathrm{NaHCO}_{3}+\mathrm{Na}_{2} \mathrm{CO}_{3}(1: 1) \\
(\mathrm{n}=6)\end{array}$} & \multicolumn{3}{|c|}{$\begin{array}{c}\mathrm{NaHCO}_{3}(100 \mu \mathrm{M}) \\
\quad(\mathbf{n}=\mathbf{3})\end{array}$} \\
\hline & Promedio & $\%$ & Duncan & Promedio & $\%$ & Duncan & Promedio & $\%$ & Duncan & Promedio & $\%$ & Duncan \\
\hline 0,0 & $23,3 \pm 2,0$ & 100 & - & $22,5 \pm 1,4$ & 100 & - & $22,7 \pm 1,2$ & 100 & - & $24,0 \pm 1,0$ & 100 & - \\
\hline 0,5 & $21,2 \pm 1,3$ & 91 & - & $20,8 \pm 0,8$ & 93 & - & $21,8 \pm 0,8$ & 96 & - & $22,7 \pm 0,6$ & 94 & - \\
\hline 1,0 & $20,0 \pm 0,9$ & 86 & - & $19,2 \pm 1,3$ & 85 & - & $19,8 \pm 1,3$ & 88 & - & $20,7 \pm 1,5$ & 86 & - \\
\hline 1,5 & $17,7 \pm 2,2$ & 76 & - & $16,5 \pm 0,8$ & 73 & - & $15,2 \pm 3,5$ & 67 & - & $15,7 \pm 0,6$ & 65 & - \\
\hline 2,0 & $12,5 \pm 2,1$ & 54 & - & $12,8 \pm 2,1$ & 57 & - & $11,5 \pm 2,9$ & 51 & - & $10,3 \pm 1,2$ & 43 & - \\
\hline 2,5 & $7,8 \pm 1,8$ & 34 & & - $\quad 9,2 \pm 1,8$ & 41 & - & $9,0 \pm 3,3$ & 40 & - & $8,0 \pm 1,0$ & 33 & - \\
\hline 3,0 & $7,0 \pm 1,7$ & 30 & & - $\quad 7,5 \pm 1,8$ & 33 & - & $7,2 \pm 2,2$ & 32 & - & $7,3 \pm 1,5$ & 31 & - \\
\hline 3,5 & $6,7 \pm 1,7$ & 29 & & A $6,8 \pm 1,6$ & 30 & A & $7,2 \pm 2,6$ & 32 & A & $7,0 \pm 1,7$ & 29 & A \\
\hline 4,0 & $6,8 \pm 1,5$ & 29 & & A $6,7 \pm 2,0$ & 30 & A & $7,7 \pm 2,7$ & 34 & A & $7,0 \pm 1,7$ & 29 & A \\
\hline 4,5 & $6,8 \pm 1,7$ & 29 & & A $7,2 \pm 1,9$ & 32 & A & $8,3 \pm 2,9$ & 37 & A & $7,0 \pm 1,7$ & 29 & A \\
\hline 5,0 & $7,3 \pm 1,5$ & 31 & & В $7,7 \pm 2,3$ & 34 & B & $10,3 \pm 1,6$ & 46 & A & $9,0 \pm 1,7$ & 38 & $\mathrm{AB}$ \\
\hline 5,5 & $8,2 \pm 1,3$ & 35 & & В $8,3 \pm 2,3$ & 37 & B & $13,2 \pm 2,0$ & 58 & A & $11,7 \pm 1,2$ & 49 & A \\
\hline 6,0 & $8,5 \pm 2,2$ & 36 & & B $9,5 \pm 3,0$ & 42 & B & $15,8 \pm 1,5$ & 70 & A & $15,7 \pm 1,2$ & 65 & A \\
\hline 6,5 & $10,2 \pm 3,1$ & 44 & B & $11,0 \pm 3,0$ & 49 & B & $18,7 \pm 1,9$ & 82 & A & $18,7 \pm 0,6$ & 78 & A \\
\hline 7,0 & $11,0 \pm 3,3$ & 47 & B & $13,7 \pm 2,9$ & 61 & B & $20,0 \pm 2,4$ & 88 & A & $21,0 \pm 1,0$ & 88 & A \\
\hline 7,5 & $13,7 \pm 3,4$ & 59 & B & $14,3 \pm 3,1$ & 64 & B & $21,2 \pm 2,2$ & 93 & A & $21,3 \pm 1,2$ & 89 & A \\
\hline 8,0 & $14,5 \pm 3,3$ & 62 & B & $16,3 \pm 4,0$ & 73 & B & $22,0 \pm 1,7$ & 97 & A & $22,7 \pm 0,6$ & 94 & A \\
\hline
\end{tabular}

inicial. El grupo testigo necesitaría 11:38:00 horas; el grupo control necesitaría 10:07:08 horas; el grupo buffer necesitaría 7:51:06 horas y el grupo bicarbonato necesitaría 7:57:06 horas. Estas predicciones indican un disminución de aproximadamente 3,5 horas la reversibilidad de la rigidez cadavérica mandibular con las dos soluciones comparadas con el tratamiento testigo y 2 horas con el control.
Tomando todos los resultados en conjunto; el carbonato, al mezclarse con el bicarbonato mejoró la velocidad de reversibilidad; pero el bicarbonato al aumentar su concentración no mostró una velocidad de reversibilidad considerable; de esta forma presentaron mejores resultados el bicarbonato de sodio a una concentración de $50 \mu \mathrm{M}$ y el buffer en concentración de $50 \mu \mathrm{M}$ de carbonato de sodio y $50 \mu \mathrm{M}$ de bicarbonato de sodio. 


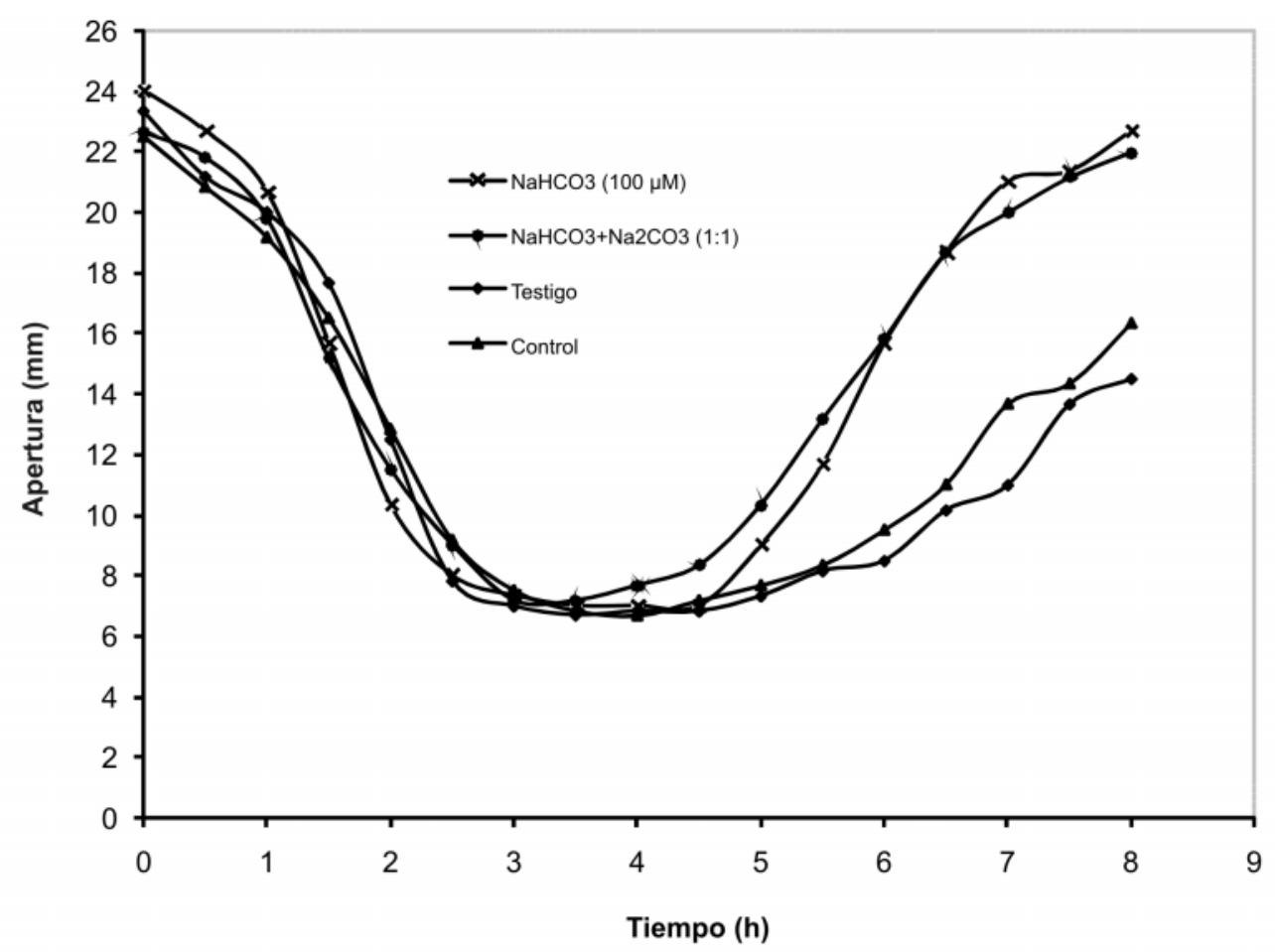

Figura 3. Comparación de sustancias segundo ensayo. Se aplicaron a las $3.5 \mathrm{~h}$ post mortem infiltraciones en los músculos masticatorios de bicarbonato de sodio $\mathrm{NaHCO}(100 \mu \mathrm{M})(\mathrm{pH}$ de 8,18$)$ y se creó una mezcla 1:1 de dos soluciones con $\mathrm{NaHCO}(50 \mu \mathrm{M})$ y carbonato de sodio $\mathrm{Na} \mathrm{CO}(50 \mu \mathrm{M})(\mathrm{pH}$ de 10$)$. Los resultados se comparan con el tratamiento testigo (sin vehículo) y con el tratamiento control (con vêhículo). Se presentan los valores promedio \pm SEM.

Tabla 5. Tabla de velocidad de reversibilidad mezclas. Como un indicador de la velocidad de reversión de la rigidez en el segundo ensayo se tomaron los valores a partir de las 5 horas hasta las 8 horas, donde se empezaron a observar diferencias significativas $(\mathrm{p}<0,05)$. Se calcularon los valores de las curvas de tendencia lineal, cuyo coeficiente de correlación lineal $\left(\mathrm{r}^{2}\right)$ fuera cercano a 1. Las pendientes de estas curvas se utilizaron arbitrariamente como indicadores de la velocidad de reversión cadavérica mandibular.

\begin{tabular}{cccc}
\hline & Pendiente $(\mathbf{m m} / \mathbf{h})$ & Intercepto $(\mathbf{m m})$ & $\mathbf{r}^{2}$ \\
\hline Testigo & 2,5000 & 5,7738 & 0,9540 \\
Control & 3,0119 & 8,0298 & 0,9763 \\
$\mathrm{NaHCO}_{3}+\mathrm{Na}_{2} \mathrm{CO}_{3}(1: 1)$ & 3,9405 & 8,3036 & 0,9537 \\
$\mathrm{NaHCO}_{3}(100 \mu \mathrm{M})$ & 4,6905 & 13,3450 & 0,9405 \\
\hline
\end{tabular}

\section{Conclusiones}

Las fases de la rigidez cadavérica en el modelo estudiado (rigor mortis), comprenden desde el inicio hasta la resolución; la fase de instauración, observada a partir de la apertura bucal en ratas, se propagó durante 2.5 horas $(150 \mathrm{~min}$ ); la fase de estado se presentó entre las 3 horas (180 min) manteniéndose constante hasta las 7 horas (420 min); a partir de las 7.5 horas (420 min) inició la fase de resolución de la rigidez cadavérica. Por medio del presente estudio se observó que la instauración de la rigidez cadavérica no se ve afectada por el peso de las ratas; cuyo comportamiento fue similar, incluso comprendiendo parte de la fase de estado hasta los 210 min. Es suficiente para la toma de evi- 
dencia bucal post mortem en el modelo de rata, utilizar una solución de $\mathrm{NaHCO}_{3}(50 \mu \mathrm{M})$ que acelera la reversibilidad cadavérica mandibular en 2 horas.

\section{Agradecimientos}

Al grupo de Neurobioquímica del Departamento de Nutrición y Bioquímica de la Facultad de Ciencias de la Pontificia Universidad Javeriana por su colaboración y apoyo en la investigación científica y recursos físicos para este estudio. Los autores agradecen a Angélica Pinzón por su colaboración en el mantenimiento de los animales de experimentación en el bioterio de la Facultad de Ciencias (PUJ).

\section{Conflicto de intereses}

Los autores declaran que no existen conflictos de intereses con el trabajo.

\section{Referencias}

1. Pretty A, Sweet DA. Look a forensic dentistry Part I: The role of teeth in the determination of human identity. British Dental Journal 001190 (7): 359-366.

2. Rothwell, B. Principles of dental identification. Dental Clinics of North America. Forensic Odontology 2001; 45 (2): 253-270.

3. Moya, P. Odontología legal y forense. Edit. Masson S.A. Barcelona, España, 1994, Cap.23; 223p.

4. Calabuig J. Medicina legal y toxicología. Ed. Nuevo Mundo. México. 1991; 155p.

5. Guyton A. Tratado de fisiología médica. Nueva Editorial Interamericana S.A., McGraw-Hill, Atrampa, México. 1991, 83.

6. Ganong W. Fisiología médica. Ed. Manual Moderno. México D.F. 2002, 155.

7. Henßgea C, Madeab B. The estimation of the time since death in the early postmortem period. Forensic Science International 2004; 144: 167-175.

8. Kobayashi m, Takeromi S. Differential rigor development in red and white muscle revealed by simultaneous measurement of tension and stiffness. Forensic Science International 2004; 140: 79-84.
9. Kobayashi M, Ikegaya H, Takase I, Hatanaka K., Sakurada K, Iwase H. Development or rigor mortis is not affected by muscle. Forensic Science International. 2001; 117: 213-219.

10. Kobayashi M, Takatori T, Nakajima M, Saka K. Iwase H, Nagao M, Niijima H, Matsuda Y. Does the sequence of onset of rigor mortis depend on the proportion of muscle fibre types and on intramuscular glycogen content?, International Journal of Legal Medicine 1999, 112: 167-171.

11. Kobayashi M, Takatori T, Nakayima M, Sakurada K. Onset of rigor mortis is earlier in red muscle than white muscle. International Journal of Legal Medicine 2000; 113: 240-243.

12. Thibodeau G, Patton K. Anatomía y fisiología, Ediciones Harcourt. Madrid, España. 2000; 320p.

13. Nakayama Y, Aoki Y, Niitsu H, Saigusa K. Forced oral opening for cadavers with rigor mortis: two approaches for the myotomy on the temporal muscles. Forensic Science. International 2001, 118 (1): 37-42.

14. Averill D. Manual of forensic odontology. Colorado, USA. 1991: 13-17.

15. Moss R., Giulian G, Greaser M. Effects of EDTA treatment upon the protein subunit composition and mechanical properties of mammalian single skeletal muscle fibers. The Journal of Cell Biology. 1983: 970-978.

16. Bjor I, Nordling K, Raub-Segall E. Inactivation of papain by antithrombin due to autolytic digestion: a model of serpin inactivation of cysteine proteinases. Biochemistry Journal. 1998; 335: 701-709.

17. Vignon C, Pellisier J. Further histochemical studies on masticatory muscles; Journal of Neurological Sciences. 1980; 45: 157-176.

18. Kobayashi, M. Why does rigor mortis progress downwards? Anil Aggrawals Internet Journal of Forensic Medicine \& Toxicology 2002; 3 (2).

19. Warris, P. Ciencia de la carne. Editorial Acribia, S.A. Zaragoza, España. 2003; Cap. 5: 102-103.

20. Smith G, Steele D. Effects of $\mathrm{pH}$ and inorganic phosphate on rigor tension in chemically skinned rat ventricular trabeculae. Journal of Physiology 1994; 478 (3): 505-512.

21. Weiner P, Pearson A. Inhibition of rigor mortis by ethylenediamine tetraacetic acid. Proceedings of the Society for Experimental Biology and Medicine 1966; 123 (1): 185-187 
22. Murray, R. Bioquímica del músculo. En: Murray R, Granner D, Mayes P. and Rodwell V. (eds.) Bioquímica de Harper. El manual moderno, México, 1997; Cap. 58, 805, 809-813p.
23. Spitz W, Steiner R, Fisher's S. Medicolegal investigation of death. Edit. Charles Thomas Publisher, Springfield, Illinois, USA. 1993; 31-32. 